\title{
Analysis on the Impacts of Governor Parameters in Isolated Power Grid
}

\author{
Xiaoqing $\mathrm{Xiao}^{1}$, Likun $\mathrm{Zheng}^{1}$, Fei $\mathrm{Li}^{2}$, a , Shiwu Xiao ${ }^{2}$ \\ ${ }^{1}$ Electric Power Research Institue of Guangdong Power Grid Corporation, \\ Guangdong, 510800, China \\ ${ }^{2}$ State Key Laboratory of Alternate Power System with Renewable Energy Sources (North China \\ Electric Power University), Beijing, 102206, China
}

aemail: 1045278574@qq.com

Keywords: Isolated Power grid; Dominant Pole; Root Locus; Primary Frequency Regulation

\begin{abstract}
In order to analysis the impact of governor parameters on the performance of primary frequency regulation, this paper built a closed loop speed regulating system model formed by turbine and generator, which is closer to the practical system. The paper simplify the high-order system in engineering by applying the conception of closed loop dominant pole. The influences of the changing of some parameters on the speed-regulating performance were intuitively present by using the performance analysis approximation method of root locus in control theory, it is pointed out the influence of the important parameters of governor control on performance of speed control system. Finally, the theory analysis is verified by the simulation results.
\end{abstract}

\section{Introduction}

The scale of modern power system is more and more large, a regional power system with another regional power system connected to each other constitute a larger system. The analysis of power system frequency modulation based on region, a large interconnected power system is divided into multiple regions, each region is seen as an equivalent synchronous generator. Frequency modulation is done mainly by the governor, turbines and generators, and governor as a key part of the speed control system, the setting and changing of its parameter have a significant impact on the power system stability and frequency modulation effect.

Literature [4-9] qualitatively points out, by the way of simulation, how the governor parameter such as dead zone and FM limiter effect the frequency dynamic characteristics. Literature [10-11] through the establishment of a FM generator mathematical model, points out the impact of generator frequency coefficient has on the system frequency response and stability. Literature [12-14] proposing a method to speed up the response speed of the turbine response, and the correctness of the method is validated by simulation. Literature [15] reproduce a power system oscillation phenomenon because of the incorrectly set of governor parameters, and point out, through the simulation, incorrectly parameter Settings lead to system instability. Most of the present study is based on simulation, and qualitatively illustrate the relationship between the governor parameters and system frequency response and stability, don't obtain, from a theoretical point of analysis, a wide range used and strong theoretical conclusions.

In this paper, a single regional speed control system model is established, and especially a fine governor model is established. The transfer function of the model is a high-order function. In order to analyze this mathematical model, a method for the use of processing and analysis of engineering based on dominant pole placement and root locus method is used. And using this way, analyzed the impact that key parameters of governor have on the speed control system dynamic performance and system stability. The correctness of the conclusion is validated by simulation.

\section{Single Regional Speed Governor System Model}

Single regional speed control system is mainly consist of governor, turbine, generator and grid. No tie-line contact with the outside power system, the single regional grid is an isolated system. 
Disturbance signal is usually a step load change. And the transfer function is a important tool to analyze the single regional speed control system performance. The physical meaning and the working principle of each part can be abstracted to transfer function. A closed loop regulating system which is represented by a mathematical model is shown in Fig.1.

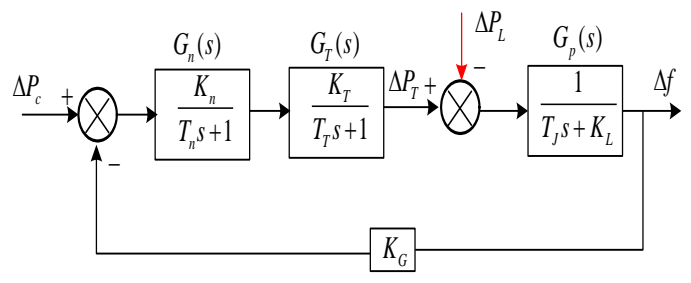

Fig.1. Frequency mathematical model of isolated system

Ggov(s) represents governor and it is general consist of measurement, integral amplifier, execute. As the input signal, the system frequency deviation is processed by speed measuring module, electro-hydraulic converter module, actuator module in turn, and the output signal is the steam valve instructions. Steam turbine receives the valve instructions, according to the instruction to open or close the valve, in this way mechanical power output is changed and changed mechanical power output changes the generator rotor speed, at last the speed of the system deviation decreases. The model of governor is shown in Fig.2. Where, $\Delta \omega$ represents speed deviation, $\mathrm{R}$ represents regulation coefficient, $K_{P 1}$ represents $P$ of load controller $P I D, K_{D 1}$ represents $D$ of load controller $\mathrm{PID}, \mathrm{K}_{\mathrm{I} 1}$ represents I of load controller PID, $\mathrm{P}_{\mathrm{CV}}$ represents valve position instruction value, $\mathrm{K}_{\mathrm{P} 2}$ represents $\mathrm{P}$ of the electro-hydraulic converter, $\mathrm{K}_{\mathrm{D} 2}$ represents $\mathrm{D}$ of the electro-hydraulic converter, $\mathrm{K}_{\mathrm{I} 2}$ represents I of the electro-hydraulic converter, VELopen represents the biggest opening speed of governor valve, VELClose represents the biggest closing speed of governor valve, $\mathrm{T}_{\mathrm{c} 1}$ represents hydraulic actuator closing time constant, $\mathrm{T}_{\mathrm{C} 2}$ represents hydraulic actuator opening time constant, PMAX represents prime mover maximum output power, PMIN represents prime mover minimum output power, $\mathrm{P}_{\mathrm{GV}}$ represents valve position value and $\mathrm{T}_{\mathrm{c}}$ represents LVDT transducer time constant.
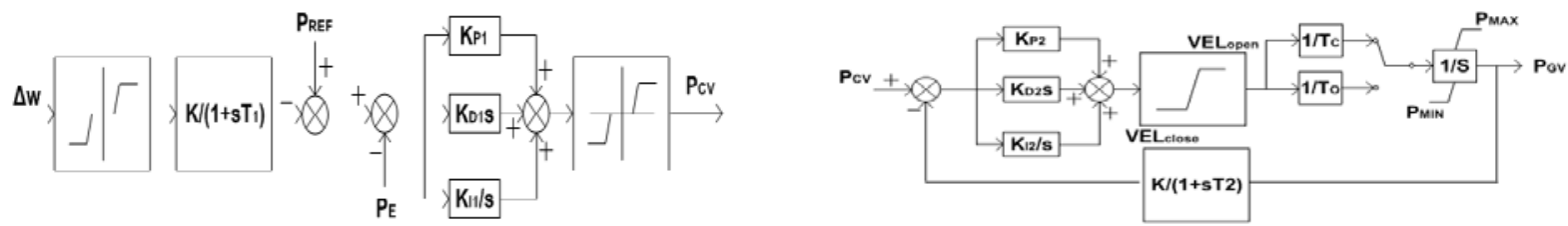

Fig.2. Classical mathematical model of governor

Ggen(s) represents generator. The analysis of power system frequency modulation based on region, a large interconnected power system is divided into multiple regions, each region is seen as an equivalent synchronous generator. For single regional speed control system, the transfer function of generator can be described as a first-order inertia module, as shown in Fig.3(a). Where, $K_{P}=1 / K_{L}$, $\mathrm{K}_{\mathrm{L}}$ represents load frequency regulation effect coefficient. $\mathrm{T}_{\mathrm{P}}=2 \mathrm{H} / \mathrm{K}_{\mathrm{L}}, \mathrm{H}$ represents generator inertia time constant.

Gtur(s) represents reheat steam turbine as shown in Fig.3(a). Where, $\mathrm{T}_{\mathrm{Ch}}$ represents the steam turbine time constant, $\mathrm{T}_{\mathrm{Rh}}$ represents reheat time constant, $\mathrm{T}_{\mathrm{CO}}$ represents low pressure pipe time constant, $\mathrm{F}_{\mathrm{HP}}$ represents high-pressure cylinder power allocation coefficient, $\mathrm{F}_{\mathrm{IP}}$ represents intermediate-pressure cylinder power allocation coefficient, $F_{L P}$ represents low-pressure cylinder power allocation coefficient, $\mathrm{P}_{\mathrm{GV}}$ represents valve position value, $\mathrm{P}_{\mathrm{M}}$ represents mechanical power output. Considering step load disturbance $\Delta P_{L}$, then $\Delta P_{L}(s)=\Delta P_{L} / \mathrm{s}$.

With the foregoing discussion, the transfer function of the whole speed control system can be got as shown in type (1). Type (1) is a high order with multiple parameters expression. Directly analysis about this transfer function will be difficult and confused. So a simplified method for the use of processing and analysis for engineering is necessary.

$$
\Delta F(\mathrm{~s})=-\frac{\operatorname{Ggen}(\mathrm{s})}{1+\operatorname{Ggov}(\mathrm{s}) \cdot \operatorname{Gtur}(\mathrm{s}) \cdot \operatorname{Ggen}(\mathrm{s})} \Delta P_{L}(\mathrm{~s})
$$

In type (1), 


$$
\begin{aligned}
& \operatorname{Ggov}(s)=\frac{1}{R} \cdot \frac{\left(K_{p 2}+\frac{K_{I 2}}{s}\right) \cdot \frac{1}{s \cdot T_{s}}}{1+\frac{1}{1+s \cdot T_{c}} \cdot\left(K_{p 2}+\frac{K_{I 2}}{s}\right) \cdot \frac{1}{s \cdot T_{s}}} \\
& \operatorname{Ggen}(s)=\frac{K_{p}}{1+s T_{p}} \\
& \operatorname{Gtur}(s)=\frac{F_{H P}}{1+s \cdot T_{c h}}+\frac{F_{I P}}{\left(1+s \cdot T_{c h}\right) \cdot\left(1+s \cdot T_{r h}\right)}+\frac{F_{L P}}{\left(1+s \cdot T_{c h}\right) \cdot\left(1+s \cdot T_{r h}\right) \cdot\left(1+s \cdot T_{c o}\right)}
\end{aligned}
$$

When the time $t \rightarrow+\infty$, then the steady-state frequency deviation $\Delta f$ can be got as following.

$$
\Delta f=\lim _{s \rightarrow 0}[s \Delta F(s)]=-\frac{K_{p}}{1+K_{p} / R} \Delta P_{L}=-\frac{\Delta P_{L}}{K_{L}+1 / R} \approx-R \Delta P_{L} \quad\left(\because K_{L} \square 1 / R\right)
$$

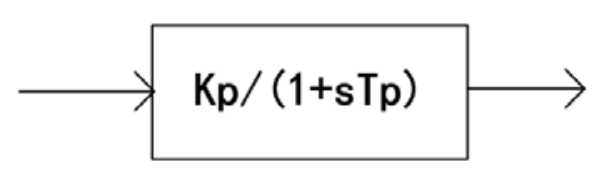

(a) Generator model

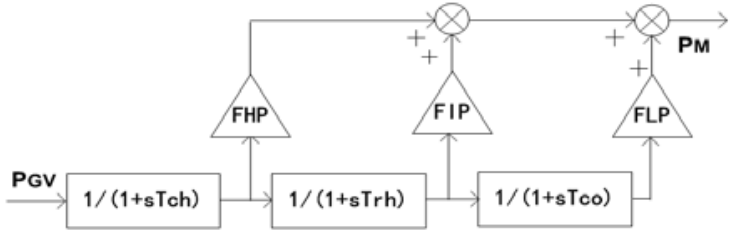

(b) Steam turbine model

Fig.3. Mathematical models of generator and steam turbine

\section{The Simplified Analysis Method based on the Theory of Automatic Control}

According to the theory of automatic control, the dynamic and steady-state performance of high-order systems is jointly determined by its closed-loop poles and zeros. Once all of the closed-loop poles, zeros and gain of a high-order is given, its dynamic and steady-state performance will be uniquely determined, and the location of the closed-loop poles in the complex plane determines the system stability margin.

In engineering, the dominant pole method is one of the principal methods to analyze higher-order system. Dominant pole method is that when all closed-loop poles of high-order system are located in the left complex plane and has a pair of dominant pole, then this high order system performance can be approximately regarded as the performance of a second-order system which is determined by the pair of above-mentioned dominant pole. High-order system dominant pole should fulfill one of the following two conditions.

1) The distance of one pair of conjugate complex poles to the imaginary axis is less than $1 / 5$ of the other poles to the imaginary axis, at the same time there is no closed loop zeros near the pair of conjugate complex poles.

2) There are no closed loop zeros near one pair of conjugate complex poles which is nearest to the imaginary axis, and there are only dipoles in 5 times the distance of the conjugate complex poles to the imaginary axis.

Root locus is that in a closed-loop system, when an adjustable parameter changes from 0 to $+\infty$, the trajectory of each closed-loop poles. It is obtained by the open-loop transfer function, and can be used to analyze the influence of variable parameters on the system performance.

\section{Performance of the Primary Frequency Regulation Response}

Use the simplified method to analyze performance of the primary frequency regulation response. According to the theory of automatic control, second order system mainly has three dynamic performance indexes.

1) One of the indexes is tr. tr is the rise time of unit step response.

2) Another of the indexes is ts. ts is the regulation time of unit step response.

3) Another of the indexes is $\sigma \% . \sigma \%$ is the overshoot of unit step response. 
The second order systems index contour map is very useful for the analysis of its dynamic performance. And the contour map is easily made by Matlab or other Mathematical analysis tools. The indexes and contour map is shown in Fig.4.

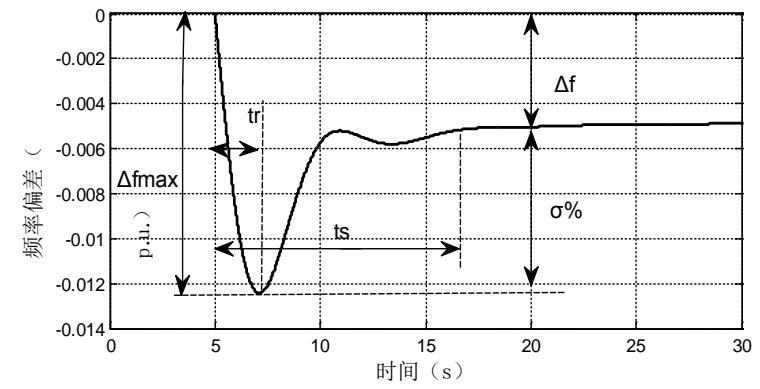

(a) Index of primary frequency regulation

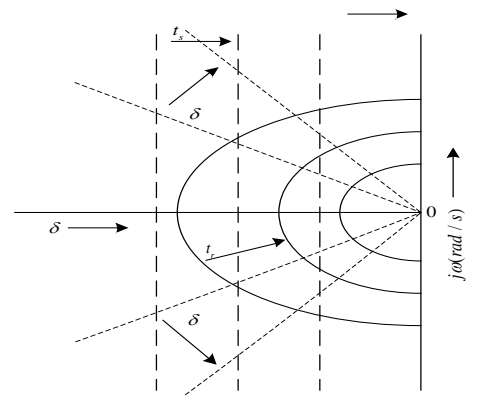

(b) contour map of tr,ts, $\sigma \%$

Fig.4.Indexes and contour map

\section{Analysis and simulation of single regional speed control system}

Regulation coefficient reflects the relationship between the change of output power and the system frequency offset. When the system frequency offset is the same, the greater the value of regulation coefficient is, the smaller the change of output power will be.

Make regulation coefficient an adjustable parameter, and take $K_{1}=1 / R$. The other parameters takes typical values, Then the open loop transfer function of the single regional speed control system is as following.

$$
G_{k 1}(s)=K_{1} \frac{264 s^{3}+1434.4 s^{2}+580.8 s+44}{80 s^{6}+812 s^{5}+1092.4 s^{4}+45664 s^{3}+11186 s^{2}+884.4 s+22}
$$

The value of hydraulic actuator time constant determines the hydraulic actuator operation speed.

Make hydraulic actuator time constant a adjustable parameter and take $K_{2}=1 / T_{s}$. The other parameters takes typical values, then the open loop transfer function of the single regional speed control system is as following.

$$
G_{k 2}(s)=K_{2} \frac{400 s^{4}+2124 s^{3}+1718.4 s^{2}+561 s+41}{8 s^{6}+441.2 s^{5}+2066.04 s^{4}+302.2 s^{3}+10 s^{2}}
$$

Make generator inertia time constant a adjustable parameter, and take $K_{3}=1 / H$. The other parameters takes typical values, then the open loop transfer function of the single regional speed control system is as following.

$$
G_{k 3}(s)=K_{3} \frac{0.04 s^{5}+2.204 s^{4}+54.22 s^{3}+1315.4 s^{2}+541 s+41}{0.04 s^{6}+2.204 s^{5}+30.22 s^{4}+105 s^{3}+20.2 s^{2}+s}
$$

Using Matlab to draw root locus of $\mathrm{K}_{1}, \mathrm{~K}_{2}$ and $\mathrm{K}_{3}$ the results are shown in Fig.5.

According to Fig 5(a), it is easily to see that the two locus with arrow is consist of dominant poles. According to the second order systems index contour map and the dominant poles locus in Fig 5(a), The greater the value of $K_{1}$ is, the bigger the overshoot $\sigma \%$ will be. At the same time, because of $|\Delta f| \approx\left|-R \Delta P_{L}\right|$, when $\Delta P_{L}$ is the same, the bigger the value of $\mathrm{K}_{1}$ is, the smaller the value of $\Delta f$ will be. Considering $\Delta f_{\max }=\Delta f(1+\delta \%)$ and the increase value of $\sigma \%$ is less than the decrease value of $\Delta f$, so when $\Delta P_{L}$ is the same, the bigger the value of $K_{1}$ is, the smaller the value of $\Delta f_{\max }$ will be. When the value of $\mathrm{K}_{1}$ is big enough, the system will be instability. 


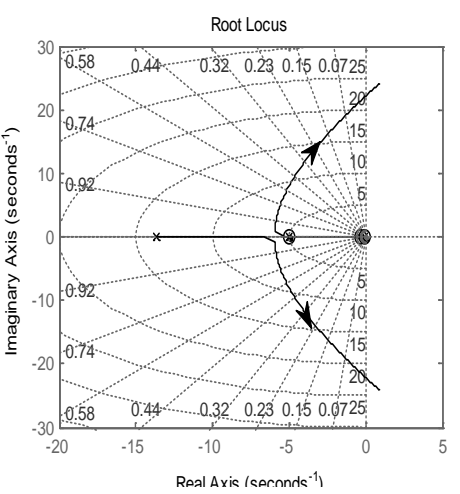

(a) Root Locus in setting of $\mathrm{K}_{1}$

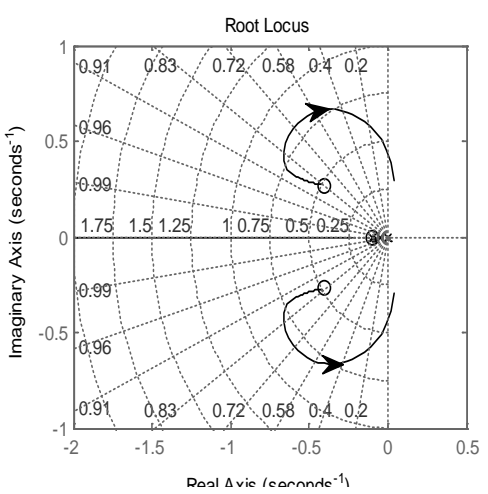

(b) Root Locus in setting of $\mathrm{K}_{2}$ $\mathrm{K}_{3}$

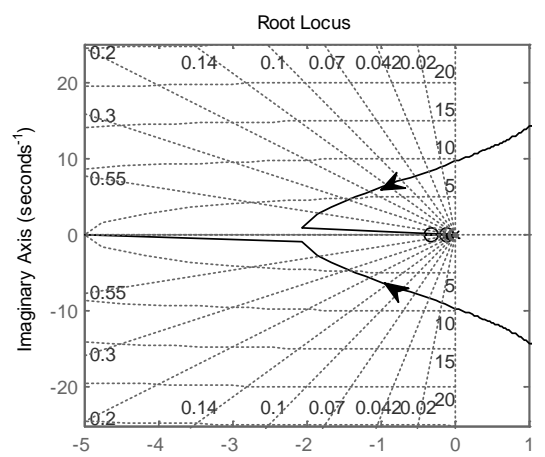

(c) Root Locus in setting of

Fig.5. Root Locus

According to Fig 5(b), the bigger the value of the Ts, the bigger the overshoot $\sigma \%$ and the regulation time ts. But when the value of Ts is small enough, the rule will be opposite. When the value of Ts is big enough, the system will be instability.

According to Fig 5(c), the Th has significant impact on rise time tr, and the bigger the value of the $T_{h}$, the bigger the rise time tr will be, but the smaller overshoot $\sigma \%$ will be.

Using the PSCAD/EMTDC to build the single regional speed control system simulation model, and the simulation results is shown in Fig.6.

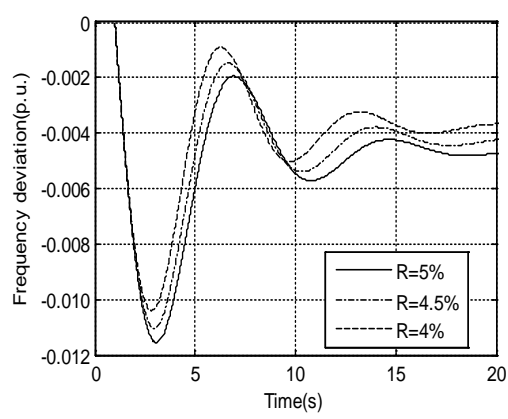

(a) In setting of $\mathrm{R}$

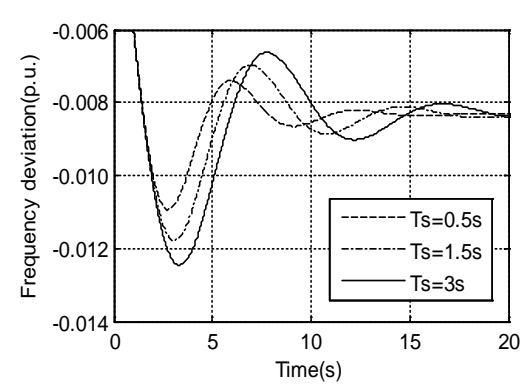

(b) In setting of Ts

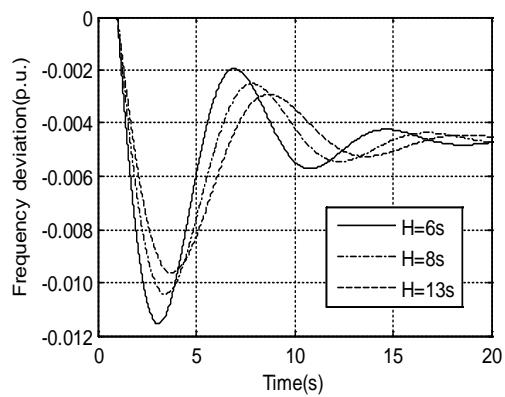

(c) In setting of $\mathrm{H}$

Fig.6. Simulation results for frequency response curve

\section{Conclusion}

In this paper, a single regional speed control system model is established and it contains governor, turbine and generator model, and a governor model. A method for the use of processing and analysis for engineering based on dominant pole placement and root locus is proposed. The method can be used to analyze dynamic performance of high-order closed-loop transfer function. Using this method, analyzed the dynamic performance impacts that key parameters(such as Regulation coefficient, Hydraulic actuator time constant, Steam turbine time constant and Generator inertia time constant)have on the frequency response of isolated power grid. And the correctness of the method is validated by simulation.

\section{Acknowledgement}

In this paper, the research was sponsored the National Basic Research Program of China (973 Program) (2012CB215200).

\section{References}

[1] Lalor G, Mullane A, O'Malley M. Frequency control and wind turbine technologies[J]. Owr Ym Ranaon on, 2005, 20(4): 1905 - 1913. 
[2] Jianming Zhang, Tianshu Bi, Hui Liu, et al. Review of frequency stability for isolated power system[J]. Power System Protection and Control,2011,39(11): 149-154.

[3] Huohua Lin,Wen Wang, Yuanyang Wei, et al. Stable operation control technologies for islanded networks in 220kV Guilin power grid[J]. Southern Power System Technology,2008,2(3):49-53.

[4] He Huang, Guanghu Xu, Chang Yu. Operation experience of isolated network in CSG during ice disaster[J]. Southern Power System Technology,2008,2(5):6-9.

[5] Jiasheng Wang, Tongtian Deng, Jingchuan Ran. Start-up and operational mode of fossil plant units in an isolated power grid[J]. Automation of Electric Power Systems,2008,32(21):102-106.

[6] Hongwei Lian, yu Zhao. Paramete optimization for frequency modulation of a unit in isolated power grid based on orthogonal test[J].Turbine Technology,2005, 47(2):108-111.

[7] Zhiping Zhou, Xinliang Sun, Chenpeng Fu,et al.Insular power system dynamicic characteristic examination research[J]. Power system Technology,2008,32(2):78-81.

[8] Peigao Zhang, Xingyuan Li, Zheng Li. Research on frequency stability and control strategy in isolated power grid [J]. Power System Protection and Control, 2012, 40(15): 143-155.

[9] Xue-Song Z, Xuan-zheng Z, You-jie M, et al. Analyze on Dynamic Frequency Characteristic of Industrial Electric Power Network under Power Situation when External Faults Occur[C]. Measuring Technology and Mechatronics Automation (ICMTMA), 2010 International Conference on. IEEE, 2010:574 - 577.

[10] Kunder P.Power system stability and control[M].New York:McGraw-Hill,1993.

[11] Wu C C , Chen N. Frequency-based method for fast-response reserve dispatch in isolated power systems[J]. IEEE Proceedings-Generation, Transmission and Distribution, 2004, 151(1): 73-77.

[12] Jianzhong Ye, Junya Zou, Fei Long, et al. Research on frequency control strategy for isolated grid operation of thermal power unit in regional power network[J]. Power System Protection and Control, 2012, 40(7):123-128.

[13] ChuanMei Zhou,Bin Sun.Study on security and stability measures of the isolated grid operation of the Guizhou power grid and area power grids[J].Power System Protection and Control,2008,36(19):29-32.

[14] Yizheng Wang, Shiying Ma, qing Wang, et al. Present status and developing trend of research on over frequency in isolated power grid[J]. Power System Technology, 2012, 40(7):165-169.

[15] Jing Zhang. Solution for DEH system in isolated network running[J].Thermal Turbine,2009, 38(1):65-67. 